
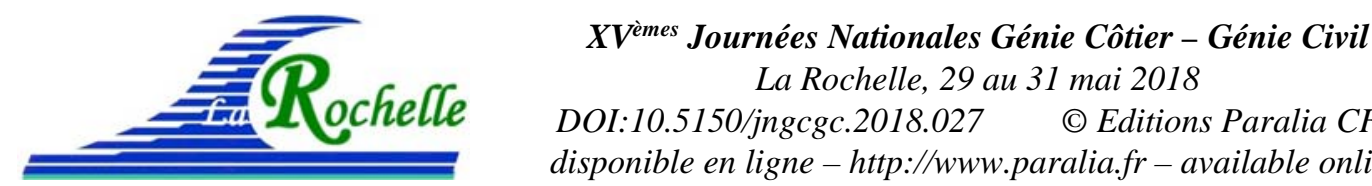

DOI:10.5150/jngcgc.2018.027 ～(C) Editions Paralia CFL

disponible en ligne - http://www.paralia.fr - available online

\title{
Suivis morphologiques et écologiques du système dunaire en réponse à la mise en place expérimentale de brèches : Truc Vert, Nouvelle-Aquitaine
}

\author{
Quentin LAPORTE-FAURET ${ }^{1}$, Bruno CASTELLE ${ }^{1}$, Richard MICHALET ${ }^{1}$, \\ Vincent MARIEU ${ }^{1}$, David ROSEBERY ${ }^{2}$, Stéphane BUJAN ${ }^{1}$, Benoit GUILLOT ${ }^{1}$ \\ 1. CNRS, Université de Bordeaux, UMR 5805 EPOC, France. \\ 2. Office National des Forêts, France.
}

\section{Résumé :}

Dans un contexte de changement climatique et d'augmentation de la pression anthropique, les littoraux sableux voient leur vulnérabilité augmenter. Historiquement, la plupart des dunes littorales ont été fixées puis gérées de manière extensive afin de protéger la forêt adjacente contre l'ensablement tout en minimisant l'érosion marine et éolienne. Cependant, les dunes littorales fixées pourraient être moins résilientes face aux perturbations naturelles et/ou anthropiques, et l'idée d'instaurer une gestion plus souple des dunes, laissant une part plus importante aux processus naturels, est de plus en plus d'actualité chez les gestionnaires des dunes côtières. Toutefois, le test expérimental de cette hypothèse n'a pas encore été effectué. Cette étude a pour objectif de mieux comprendre les interactions s'exerçant entre les processus physiques et biologiques face aux perturbations dans les systèmes dunaires côtiers. Pour cela, une expérimentation originale a été mise en place sur une longueur de $4 \mathrm{~km}$ de cordon dunaire sur la plage du Truc Vert en Gironde (Aquitaine), où huit larges brèches ont été creusées en décembre 2017 à différents endroits stratégiques du système dunaire. Son évolution est suivie par (1) des relevés topographiques du système plage-dune par stéréophotogrammétrie d'images drone et (2) des mesures de composition et d'abondance de la végétation effectuées le long de transects cross-shore situés dans l'alignement des brèches expérimentales et en situation témoin. Les premiers résultats montrent une évolution significative de la morphologie des trous après les premières tempêtes hivernales de 2017/2018, avec toutefois des différences importantes en fonction de leurs emplacements dans le système plage-dune. Les mesures de la végétation dunaire avant creusement des trous montrent une importante discrimination des espèces en fonction de leur capacité à résister à l'ensablement.

Mots-clés : Géomorphologie, Dune littorale, Végétation côtière, Caoudeyre, Résilience, Expérimentation

\section{Introduction}

Les dunes littorales procurent d'importants services écosystémiques tout en formant une barrière naturelle efficace contre les submersions marines et les vagues de tempêtes. 


\section{Thème 2 - Dynamique sédimentaire}

Elles se sont développées dans le monde le long des côtes possédant à la fois un apport suffisant en sable, des vents côtiers dominants et la présence de végétation et/ou d'autres obstructions piégeant le sable transporté par le vent (NORDSTORM, 2015). Dans ces milieux, de nombreux processus, biotiques et abiotiques, interagissent entre eux sur une vaste gamme d'échelles spatio-temporelles. Les vagues peuvent causer une érosion drastique du littoral lors d'événements de tempêtes (CASTELLE et al, 2015) ou contribuer à son rétablissement en ramenant le sable vers la côte lors de faibles conditions de houle (FERNANDEZ-MORA et al, 2015) tandis que les processus éoliens déplacent le sable depuis la plage vers la dune (HESP \& WALKER, 2013). Enfin, la végétation dunaire limite l'érosion éolienne en captant le sable par ses parties aériennes et en le fixant par ses racines et rhizomes. Les interactions entre ces processus peuvent mener au développement de caoudeyres, formées par l'érosion éolienne, dans les dunes littorales. Elles apparaissent sous forme de dépression en soucoupe, large et peu profonde, ou en forme de V, plus profonde avec des murs d'érosion raides (HESP, 2002). Le vent est topographiquement accéléré au sein des caoudeyres, érode la base du bassin de déflation et transporte les sédiments dans le lobe de dépôt ou au-delà, favorisant ainsi les transferts sédimentaires depuis la plage vers l'arrière dune (HESP \& WALKER, 2013). La plantation de végétaux pour stabiliser les dunes, tout en augmentant leur volume, est une pratique courante dans la gestion de ces systèmes depuis le XIXème siècle. Elle était effectuée à l'origine pour protéger la forêt contre l'ensablement. Cependant, la stabilisation intensive des dunes réduit leur naturalité et l'impact de cette stabilisation sur la biodiversité est encore peu connu. Depuis les années 1990, cette stratégie de fixation des dunes littorales semble évoluer vers une gestion plus souple en mettant en avant les processus naturels afin de renforcer la dynamique des habitats naturels. En effet, certains gestionnaires des systèmes dunaires avancent l'hypothèse que des dunes en libre évolution seraient plus résilientes face aux perturbations naturelles et/ou anthropiques, mais cette hypothèse reste à vérifier et dépend certainement du type de dune. Cette étude a pour objectif de mieux comprendre les interactions s'exerçant entre les processus physiques et biologiques face aux perturbations dans les systèmes dunaires littoraux de la côte aquitaine.

\section{Matériel et méthodes}

\subsection{Site d'étude}

La plage du Truc Vert (Sud-Ouest de la France) (figure 1.a) est une plage sableuse ouverte de haute énergie, méso- à macro-tidale, avec un marnage moyen de 3,7 m lors des marées de vive eau et un trait de côte relativement stable ces 65 dernières années (CASTELLE et al., 2018).

La plage du Truc Vert est bordée par un important cordon dunaire éolien de près de 200 ans, atteignant des hauteurs de 20 à $25 \mathrm{~m}$ au-dessus de niveau de la mer et des largueurs 


\section{XVèmes Journées Nationales Génie Côtier - Génie Civil \\ La Rochelle, 29 au 31 mai 2018}

de $250 \mathrm{~m}$. Depuis les années 1960, et après avoir subi d'importants dégâts lors des hivers au début du siècle et durant la seconde guerre mondiale (BARRERE, 1992), l'Office National des Forêt (ONF) a entrepris d'importants travaux de profilage des dunes et de plantation d'espèces végétales dans le but de rénover le système dunaire. Depuis, la dune est toujours entretenue notamment pour prévenir de la formation de caoudeyres. Au niveau de la végétation, on distingue de la plage à la forêt quatre types de dunes, la dune embryonnaire dominée par Elymus farctus et Euphorbia paralias, la dune blanche dominée par Ammophilia arenaria, la dune grise dominée Helichrysum stoechas et la lette grise dominée également par Helichrysum stoechas mais où les lichens deviennent très abondants (FOREY et al., 2008).
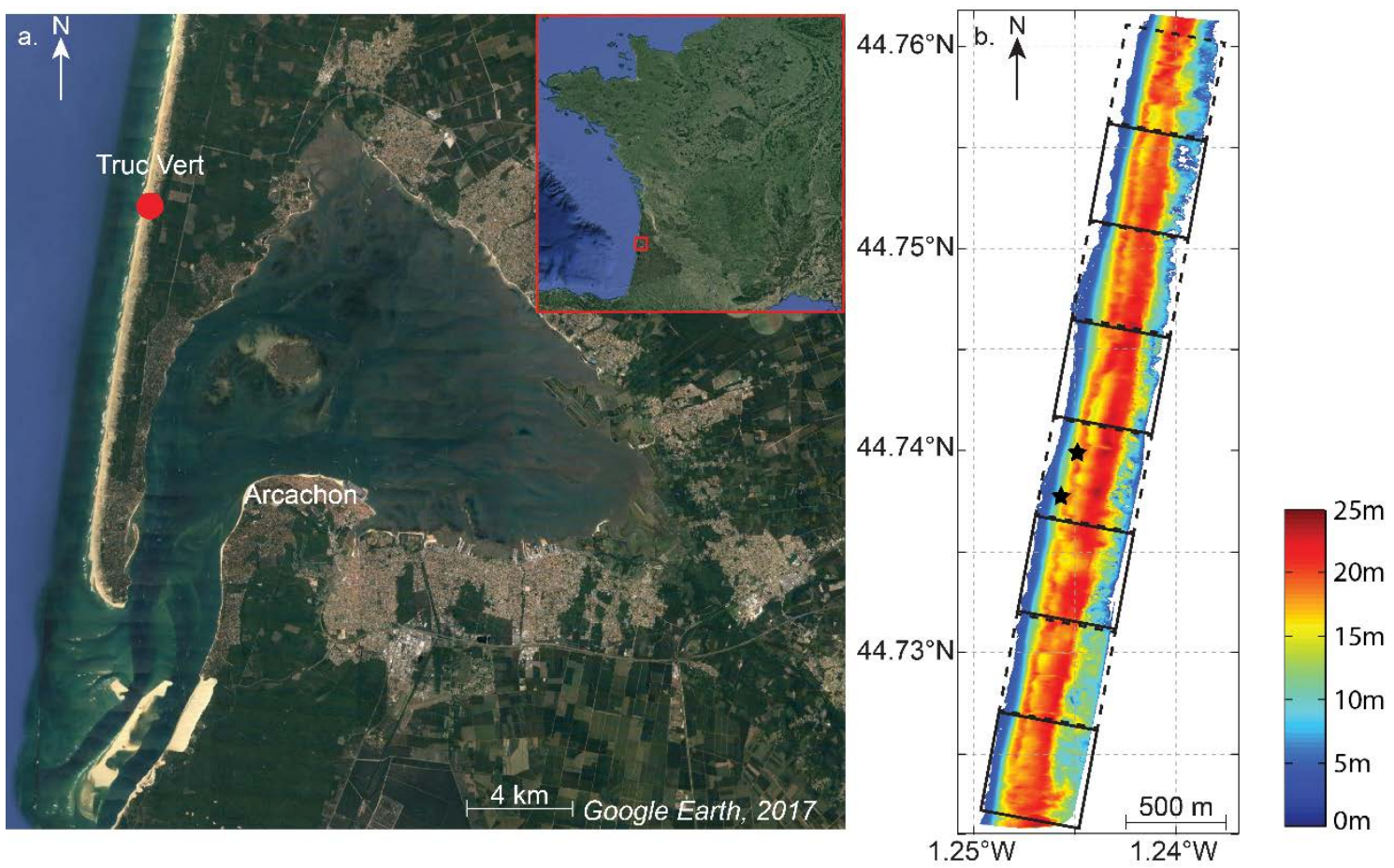

Figure 1. (a) Carte de la localisation de la plage du Truc Vert. (b) Modèle numérique de terrain de la plage du Truc Vert avec les zones où les brèches expérimentales ont été creusées (carrés en pointillés) et les zones à gestion habituelle servant de zones témoins (carrés en traits continus). Les étoiles représentent l'emplacement des trou 3 et 4 de la figure 2.b et 2.c.

\subsection{Protocole expérimental}

Le site atelier est un tronçon de $4 \mathrm{~km}$ de la plage du Truc Vert, subdivisé en huit sections de $500 \mathrm{~m}$ (figure 1.b). Dans quatre de ces huit sections, deux larges brèches ont été creusées, afin de simuler des caoudeyres, une à la limite entre la dune embryonnaire et la dune blanche, appelée trou Ouest (figure 2.b) et l'autre au milieu de la dune blanche, appelée trou Est (figure 2.c). Le sable récolté a été déposé et aplani au nord du 


\section{Thème 2 - Dynamique sédimentaire}

trou. Les quatre autres sections servent quant à elles de témoin. Ces brèches ont été creusées avec des dimensions horizontales similaires aux siffle-vents naturellement présents dans la zone d'étude. Au total, huit trous ont été réalisés, numérotés successivement de 1 à 8 du sud vers le nord. Les trous $1,3,5$ et 7 correspondent aux trous Ouest et les trous 2, 4, 6 et 8 correspondent aux trous Est. Les volumes de sables excavés pour former ces trous sont présentés dans le tableau 1.

Tableau 1. Volumes $\left(\mathrm{m}^{3}\right)$ de sable excavés pour les huit trous.

\begin{tabular}{|c|c|c|c|c|c|c|c|c|}
\hline Trou & Trou 1 & Trou 2 & Trou 3 & Trou 4 & Trou 5 & Trou 6 & Trou 7 & Trou 8 \\
\hline Volume $\left(\mathrm{m}^{3}\right)$ & 27,93 & 96,69 & 70,56 & 62,44 & 78,94 & 52,08 & 14,48 & 50,58 \\
\hline
\end{tabular}
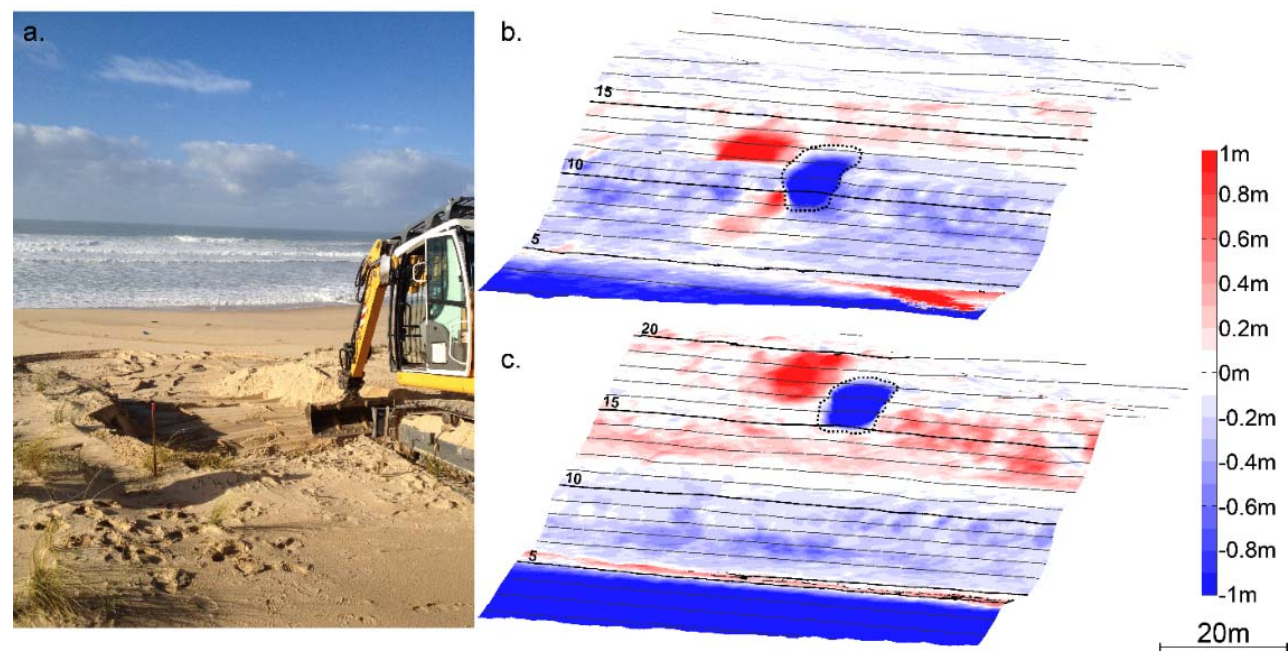

Figure 2. (a) Photographie des travaux effectués sur la plage du Truc Vert le 12.12.17, (b) Différence entre les modèles numériques de terrain avant les travaux, le 06.12.17, et après les travaux, le 19.12.17, dans la dune embryonnaire (trou 3) et (c) dans la dune blanche (trou 4). La bordure des trous est représentée en pointillés.

\subsection{Suivis géomorphologiques}

Le suivi de la géomorphologie du système plage-dune du Truc Vert est réalisé par un drone quadri copter DJI Phantom 2 équipé d'une caméra GoPro 4 Black Edition fixée sur une nacelle de stabilisation 3 axes DJI Zenmuse H3-3D. La topographie du système est réalisée par stéréophotogrammétrie d'images, à l'aide de points de contrôle permanents, pour obtenir des modèles numériques de terrain d'une résolution de $0,1 \mathrm{~m}$. L'approche utilisée est détaillée dans GUILLOT et al. (sous presse).

\subsection{Suivis de la végétation}

Le suivi saisonnier de la végétation dunaire est réalisé le long de vingt transects, respectivement numérotés de 1 à $20 \mathrm{du}$ sud vers le nord, s'étalant de la plage à la dune 


\section{XVèmes Journées Nationales Génie Côtier - Génie Civil \\ La Rochelle, 29 au 31 mai 2018}

grise. Deux cents soixante relevés quantitatifs sont effectués à l'aide d'un quadra couvrant une surface de $1 \mathrm{~m}^{2}$ et quadrillé de $0,2 \mathrm{~m}$ par $0,2 \mathrm{~m}$. Les quadras ont été localisés dans chaque transect principalement au niveau des différents écotones comme indiqué dans la figure 3. Ces données sont ensuite traitées par analyse factorielle des correspondances avec le logiciel R.

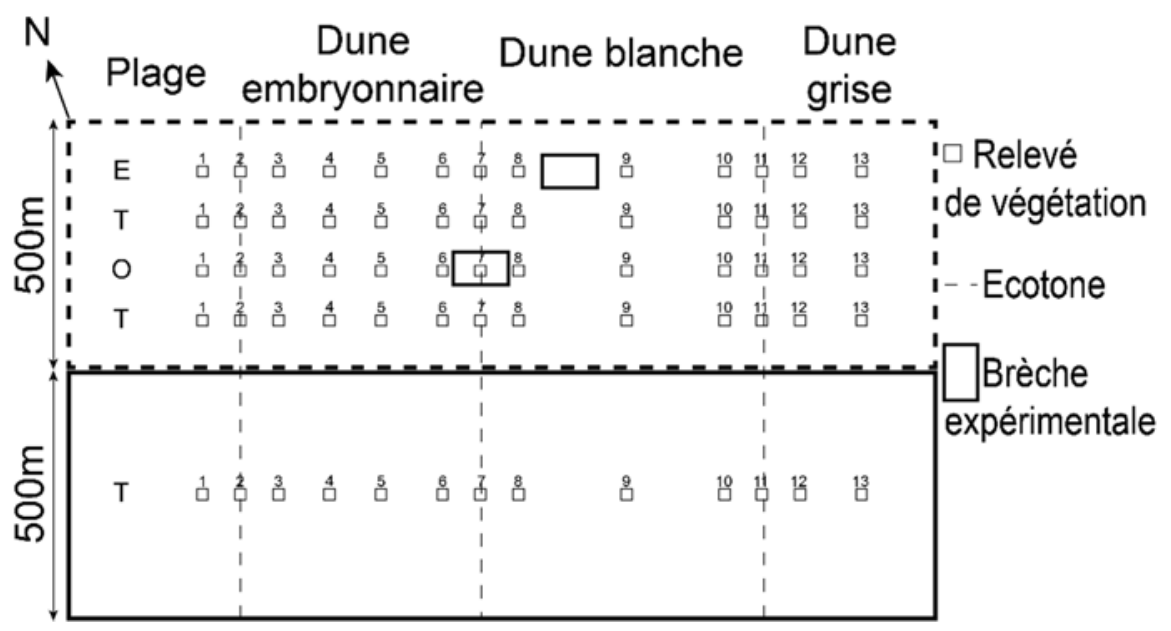

Figure 3. Positionnement des relevés de végétation dans une zone témoin (rectangle en trait plein) et une zone expérimentale contenant deux brèches artificielles (rectangle en pointillés). En face des transects, les lettres $T$, E et $O$ représentent respectivement les transects témoins, avec un trou Est et avec un trou Ouest

\section{Résultats}

\subsection{Evolution topographique du système dunaire}

Les premiers résultats de cette expérimentation obtenus durant le mois suivant le façonnement des trous du 12 décembre 2017 sont présentés dans la figure 4. Le site d'étude montre une tendance à l'accrétion sur le haut de plage, la face océanique de la dune blanche au centre du système, la crête et l'arrière de la dune blanche avec des valeurs de $0,3 \mathrm{~m}$ à $0,5 \mathrm{~m}$. En revanche, la dune embryonnaire et les faces océaniques de la dune blanche au nord et au sud du système se démarquent par d'importants taux d'érosion verticale généralement compris entre 0,4 et $0,5 \mathrm{~m}$. La dune grise semble peu affectée, avec seulement des petites valeurs accrétion et érosion, autour de $0,1 \mathrm{~m}$, respectivement au sud et au centre du site d'étude (figure 4.a, b, c, d, e, f, g, h). Les trous $1,3,5$ et 7 présentent des taux d'érosion respectifs d'environ $0,2 \mathrm{~m}, 0,4 \mathrm{~m}, 0,5 \mathrm{~m}$ et $0,3 \mathrm{~m}$ traduisant ainsi un élargissement et un approfondissement (figure 4.a, c, e, g). Le trou 2 affiche une érosion de ses parois d'environ 0,2 à $0,3 \mathrm{~m}$ (figure 4.b), tandis que les trous 4,6 et 8 présentent des taux d'accrétion respectifs d'environ $0,4 \mathrm{~m}, 0,5 \mathrm{~m}$ et $0,3 \mathrm{~m}$ (figure $4 . \mathrm{d}, \mathrm{f}, \mathrm{h}$ ). 


\section{Thème 2 - Dynamique sédimentaire}

\subsection{Etat de la végétation avant perturbation}

La figure 5 montre l'abondance moyenne des espèces végétales sur les 20 transects, en fonction de la distance au trait de côte lors des premiers relevés de la végétation effectués en décembre 2017, avant la mise en place des brèches artificielles dans le système. Les différents espaces du système plage-dune ont été délimités par la distance moyenne entre les écotones et le trait de côte. Les espèces végétales se répartissent distinctement en fonction des zones dans la dune avec la présence d'Elymus farctus et d'Euphorbia paralias dans la dune embryonnaire, avec des valeurs d'abondances atteignant $10 \%$ et $17 \%$, d'Ammophila arenaria et de Galium arenarium dans la dune blanche, avec des valeurs d'abondances atteignant $51 \%$ et $37 \%$, et d'Helichrysum stoechas dans la dune grise, avec des valeurs d'abondances de 37\%. Sur ce même jeu de données, une analyse factorielle des correspondances a été réalisée (figure 6). Chaque numéro correspond à un relevé effectué à l'aide d'un quadra. Plus les relevés sont proches, plus les espèces végétales les composant sont similaires. Plus les relevés sont proches d'une espèce végétale, plus ils sont influencés par cette dernière. Les données se répartissent en forme de croissant, aussi appelé effet Guttman (GUTTMAN, 1941) étant donné l'existence sur les deux axes d'un gradient majeur unique observable d'une extrémité à l'autre du croissant en relation avec la position des quadras sur les transects (éloignement de la plage).

\section{Discussion et conclusions}

En seulement un peu plus d'un mois de suivi, le système plage-dune du Truc Vert a montré des évolutions morphologiques significatives dans et autour des brèches expérimentales. Durant cette période, le littoral environnant a été frappé par trois événements de tempêtes majeurs, les tempêtes Bruno, Carmen et Eleanor caractérisées par des rafales de vent supérieures à $120 \mathrm{~km} / \mathrm{h}$. Le haut de plage montre une accrétion importante sur toute la zone, suivie par une érosion constante de la dune embryonnaire. Les trous Ouest se démarquent par les valeurs d'érosion les plus importantes dans le système. Cela indique que le vent a bien été topographiquement forcé par ces perturbations, et que par rétroaction positive entre les flux éoliens et l'évolution de la topographie, la caoudeyre a continué à se développer. L'approfondissement de celles-ci ainsi que l'érosion de leurs parois semblent montrer une évolution morphologique des brèches de la dune embryonnaire vers des caoudeyres en forme de $\mathrm{V}$.

Les sédiments ainsi transportés par le vent depuis la dune embryonnaire peuvent expliquer les importants taux d'accrétion qui se retrouvent sur la crête et à l'arrière de la dune blanche, avec une dune blanche reculant lentement vers les terres. En effet, les dunes perturbent l'écoulement de l'air et provoquent une accélération de ce dernier sur la pente face au vent, caractérisé par une érosion préférentielle, et une séparation et décélération de l'écoulement à la crête et sur la pente sous le vent de la dune, favorisant le dépôt sédimentaire (WALKER \& HESP, 2013). Dans ce contexte, l'évolution des 


\section{XVèmes Journées Nationales Génie Côtier - Génie Civil \\ La Rochelle, 29 au 31 mai 2018}

trous Est n'est pas homogène. Trois des quatre trous creusés en haut de dune blanche montrent une tendance au dépôt, à travers une rétroaction négative, avec parfois des variations au sein même du trou, et des évolutions morphologiques limitées autour du trou. Il est ainsi possible de penser que le dimensionnement initial des brèches expérimentales dans le haut de dune n'était pas adéquat. En ce qui concerne la végétation dunaire, ces premiers résultats vont dans le sens des travaux de FOREY et al. (2008) qui ont mis en évidence que le facteur dominant de la composition végétale des dunes est le gradient d'ensablement. L'ensablement agit comme une importante perturbation supprimant les espèces non adaptées, conformément au modèle de GRIME (1974). Par ailleurs les niches des espèces le long de ce gradient majeur sont clairement indépendantes et non pas emboitées conformément au modèle de WHITTAKER (1956), montrant le caractère individualiste des espèces dans ce système. Ainsi, la mise en place de perturbations mécaniques expérimentales dans le système plage-dune du Truc Vert a permis de mettre en évidence une réponse morphologique différenciée du système à cette perturbation, avec une érosion des trous creusés dans la dune embryonnaire et une accrétion des trous creusés dans la dune blanche. L'évolution de l'impact de trous sur la morphologie de la dune et la dynamique de végétation sera étudiée dans les 3 années futures. Plusieurs mesures environnementales supplémentaires seront mises en place, telles que la mesure de l'ensablement au niveau des quadras, le taux de décomposition de la matière organique ou encore le potentiel abrasif du vent, afin de mieux comprendre les facteurs directs déterminant la réponse des espèces végétales aux perturbations dans la dune. 


\section{Thème 2 - Dynamique sédimentaire}

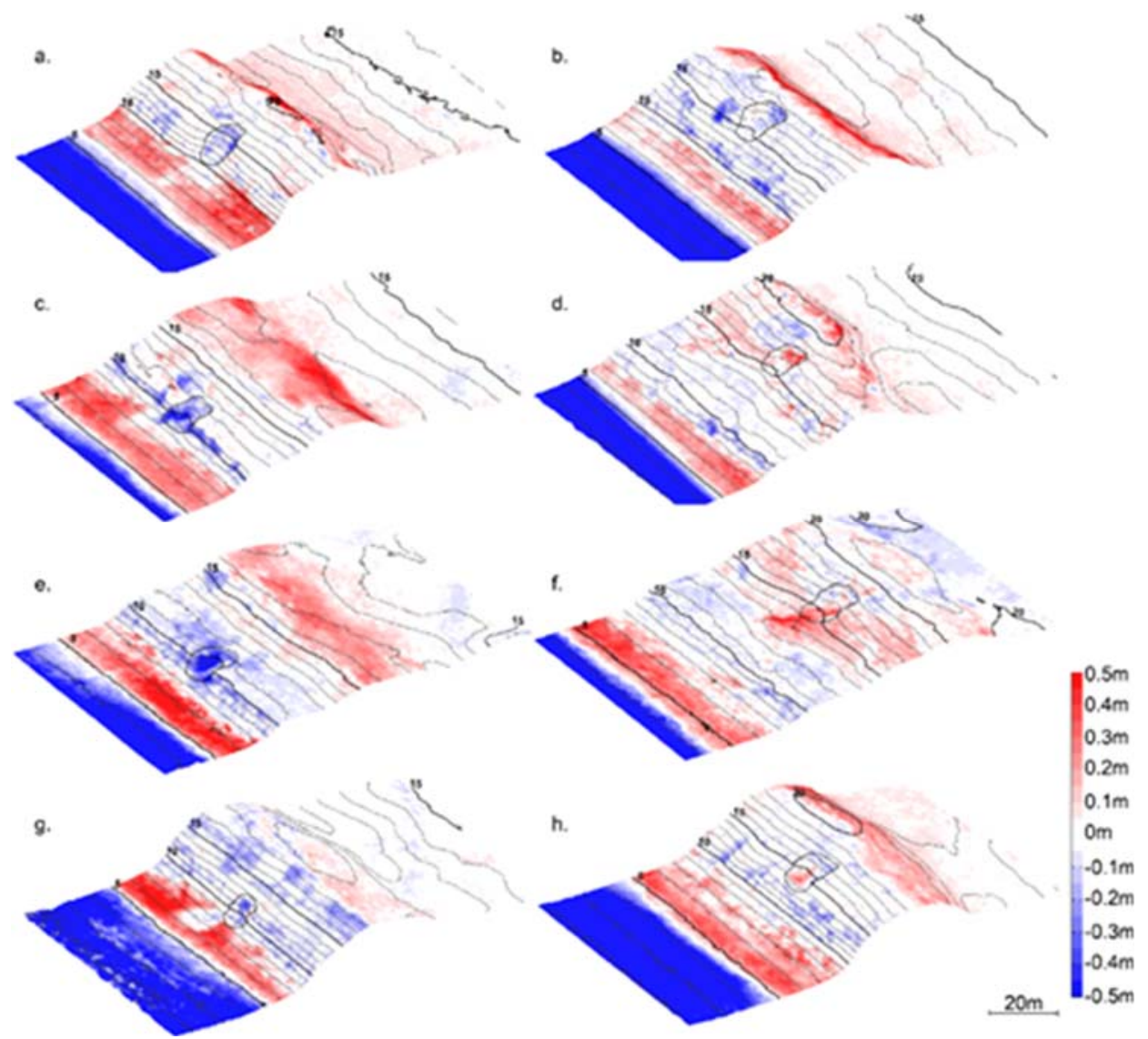

Figure 4. Différence entre les modèles numériques de terrain des zones de la dune autour des trous, entre le 19.12.17 et le 23.01.18, avec les trous (a) 1, (c) 3, (e) 5 et (g) 7 dans la dune embryonnaire et (b) 2, (d) 4, (f) 6 et (h) 8 dans la dune blanche. La bordure des trous est représentée en pointillés.

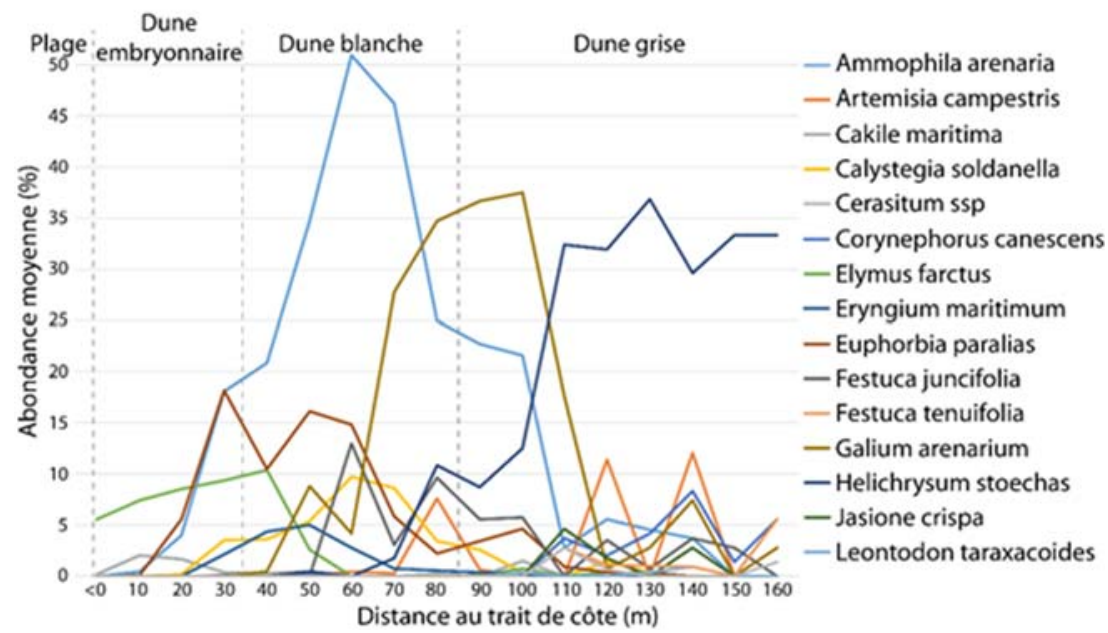

Figure 5. Abondance moyenne des espèces végétales du système plage-dune du Truc Vert sur les 20 transects (\%) en fonction de la distance au trait de côte (m) avant la mise en place des perturbations mécaniques. 


\section{XVèmes Journées Nationales Génie Côtier - Génie Civil \\ La Rochelle, 29 au 31 mai 2018}

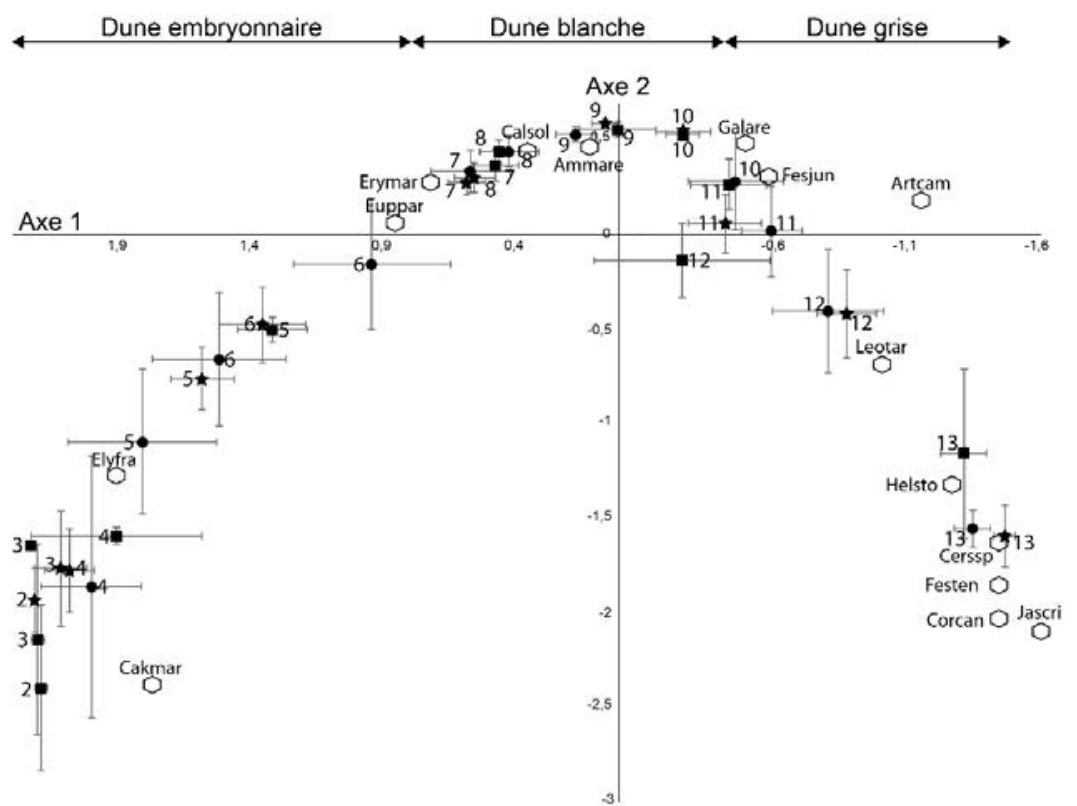

Figure 6. Analyse factorielle des correspondances sur les abondances moyennes des espèces végétales avant la mise en place des perturbations mécaniques. Les étoiles, ronds et carrés correspondent respectivement aux transects témoins, Ouest et Est. La numérotation correspond à l'emplacement du relevé.

\section{Références bibliographiques}

BARRERE P. (1992). Dynamics and management of the coastal dunes of the Landes, Gascony, France, in: Carter, R.W.G. et al. (Ed.) Coastal dunes: geomorphology, ecology and management for conservation: Proceedings of the 3rd European Dune Congress Galway, Ireland, 17-21 June 1992. pp. 25-32.

CASTELLE B., MARIEU V., BUJAN S., SPLINTER K.D., ROBINET A., SENECHAL N., FERREIRA S. (2015). Impact of the winter 2013-2014 series of severe western Europe storms on a double-barred sandy coast: beach and dune erosion and megacusp embayments. Geomorphology, Vol. 238, pp 135-148.

doi: 10.1016/j.geomorph.2015.03.006

CASTElle B., GUILlOT B., MARIEU V., CHAUMILlON E., HANQUIEZ V., BUJAN S., POPPESCHI C. (2018). Spatial and temporal patterns of shoreline change of a 280-km high-energy disrupted sandy coast from 1950 to 2014: SW France. Estuarine, Coastal and Shelf Science, Vol. 200, pp 212-223. https://doi.org/10.1016/j.ecss.2017.11.005

FERNANDEZ-MORA A., CALVETE D., FALQUES A., DE SWART H.E. (2015). Onshore sandbar migration in the surf zone: new insights into the wave-induced sediment transport mechanisms. Geophys. Res. Lett., Vol. 42 (8), pp 2869-2877. doi : $\underline{10.1002 / 2014 \mathrm{~g} 1063004}$ 


\section{Thème 2 - Dynamique sédimentaire}

FOREY E., CHAPELET B., VITASSE Y., TILQUIN M., TOUZARD B., MICHALET R. (2008). The relative importance of disturbance and environmental stress at local and regional scales in French coastal sand dunes. Journal of Vegetation Science, Vol. 19, pp 493-502. https://doi.org/10.3170/2008-8-18392

GUILLOT B., CASTELlE B., MARIEU V., BUJAN S., ROSEBERY D. UAV Monitoring of 3-year foredune partial recovery from a severe winter: Truc Vert beach, SW France. Journal of Coastal Research, Issue Special No. 85, (sous presse).

GUTTMAN L. (1941). The quantification of a class of attributes: a theory and method of scale construction. In: The Prediction of Personal Adjustment. HORST P., et al (Ed), Social Science Research Council, New York.

GRIME J.P. (1974). Vegetation classification by reference to strategies. Nature, Vol. 250, Issue 5461, pp 26-31. doi:10.1038/250026a0

HESP P.A. (2002). Foredunes and blowouts: initiation, geomorphology and dynamics. Geomorphology, Vol. 48, pp 245-268. https://doi.org/10.1016/S0169-555X(02)00184-8

HESP P.A., WALKER I.J. (2013). Aeolian environments: coastal dunes. In: Shroder J., Lancaster N., Sherman D.J., Baas A.C.W. (Eds.), Treatrise on Geomorphology. Aeolian Geomorphology, Vol. 11. Academic Press, San Diego, pp. 109-133.

NORDSTROM K.F. (2015). Coastal dunes. In: Masselink G., Gehrels R. (Ed.), Coastal Environments and Global Change. John Wiley \& Sons, Ltd., pp 178-193.

WALKER I.J., HESP P.A. (2013). Fundamentals of aeolian sediment transport: airflow over dunes. In: Shroder J., Lancaster N., Sherman D.J., Baas A.C.W. (Eds.), Treatise on Geomorphology, Aeolian Geomorphology, Academic Press, San Diego, CA, vol.11, pp.109-133.

WHITTAKER R.H. (1956). Vegetation of the Great Smoky Mountains. Ecol. Monogr., Vol. 26, pp 1-80. 